\title{
Design and fabrication of low dielectric constant Poly(arylene ether sulfone) film containing cyclohexane group
}

Gang Zhang ${ }^{1}$, Dongsheng $\mathrm{Li}^{2}$, Guangming Yan ${ }^{1}$, Han Wang ${ }^{3}$, Yu Zhang ${ }^{3}$, Zhefu $\mathrm{Wu}^{3}$, Suilin Liu ${ }^{1}$, Xiaojun Wang ${ }^{1 *}$, Jie Yang ${ }^{1}$

1 Institute of Materials Science and Technology, Analysis and testing center, Sichuan University,

Chengdu 610064, P. R. China

2 Shaanxi Engineering Research Center of Special Sealing Technology, Xi'an Aerospace propulsion Institute, Xi'an 710100, P. R. China

3 College of polymer materials science and engineering of Sichuan University, Chengdu 610064,

P. R. China

Corresponding email: wangxj@scu.edu.cn 


\section{Supporting Information}

\section{Synthesis of 4, 4'-(cyclohexane-1,1-diyl)bis(2-methoxyphenol) (CDBMP)}

Chemical information of CDBMP in the spectrum of FT-IR and ${ }^{1} \mathrm{H}-\mathrm{NMR}$ was provided in the following:

FT-IR (KBr, cm $\left.{ }^{-1}\right): 3407(-\mathrm{OH}), 3028$ (C-H aromatic ring), 2933, $2865\left(-\mathrm{CH}_{3},-\mathrm{CH}_{2}-\right)$, 1611, 1516 (C=C aromatic ring), 1143 (-O-), NMR [400 MHz, deuterated dimethyl sulfoxide (DMSO-d6)/tetramethylsilane, ppm]: 1.455-1.465 (m, 6H, H1-H2), 2.165 (s, 4H, H3), 3.696 (s, 6H, H4), 6.858-6.868 (d, 4H, H5-H6), 6.798 (s, 2H, H7), 8.683 (s, $2 \mathrm{H},-\mathrm{OH})$.

\section{The FT-IR and ${ }^{1} \mathrm{H}-\mathrm{NMR}$ results of $\mathrm{PES}-\mathrm{OCH}_{3}$}

FT-IR (KBr, cm $\left.{ }^{-1}\right): 3075$ (C-H aromatic ring), 2940, $2859\left(-\mathrm{CH}_{3},-\mathrm{CH}_{2}-\right), 1598,1510$ $\left(\mathrm{C}=\mathrm{C}\right.$ aromatic ring), 1320, $1150\left(-\mathrm{SO}_{2}-\right), 1110(-\mathrm{O}-)$; NMR [400 MHz, deuterated dimethyl sulfoxide (DMSO-d6)/tetramethylsilane, ppm]: 1.598-1.626 (d, 6H, H1-H2), 2.275 (s, 4H, H3), 3.696 (s, 6H, H4), 6.866-6.955 (m, 10H, H5-H8), 7.788-7.810 (d, 4H, H9).

\section{The FT-IR and ${ }^{1} \mathrm{H}-\mathrm{NMR}$ results of PES-OH and PES-Boc}

PES-OH: FT-IR (KBr, cm $\left.{ }^{-1}\right), 3421(-\mathrm{OH}), 3075$ (C-H aromatic ring), 2920, 2859 ($\left.\mathrm{CH}_{3},-\mathrm{CH}_{2-}\right), 1584,1489$ (C=C aromatic ring), 1313, $1143\left(-\mathrm{SO}_{2}-\right), 1090$ (-O-); NMR [400 MHz, deuterated dimethyl sulfoxide (DMSO-d6)/tetramethylsilane, ppm]: 1.474 (s, 6H, H1-H2), 2.161 (s, 4H, H3), 6.794-6.991 (m, 10H, H4-H7), 7.799-7.842 (d, 4H, H8), 9.548 (s, 2H, -OH).

PES-Boc: FT-IR (KBr, cm$\left.{ }^{-1}\right), 3069$ (C-H aromatic ring), 2979, 2934, $2862\left(-\mathrm{CH}_{3}\right.$, - 
$\left.\mathrm{CH}_{2}-\right), 1763(\mathrm{C}=\mathrm{O}), 1588,1491(\mathrm{C}=\mathrm{C}$ aromatic ring $), 1326,1147\left(-\mathrm{SO}_{2^{-}}\right), 1147,1106$ (-O-); NMR [400 MHz, deuterated Chloroform ( $\left.\mathrm{CDCl}_{3}\right)$ /tetramethylsilane, ppm]: 1.37 (s, 18H, H1), 1.54-1.56 (m, 6H, H2-H3), 2.24 (s, 4H, H4), 6.94-6.96 (d, 2H, H5), 7.007.02 (d, 4H, H6), 7.10-7.11 (d, 2H, H7), 7.17 (s, 2H, H8), 7.82-7.85 (d, 4H, H9).
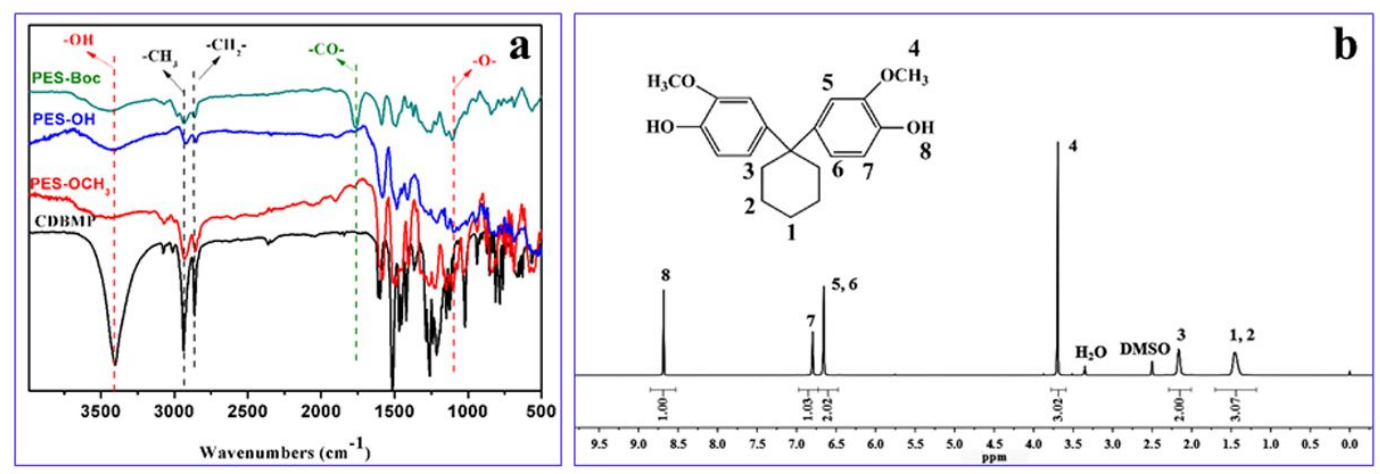

Figure S1 Chemical structure of monomer and polymers, a. the FT-IR spectra of CDBMP, PES-OCH ${ }_{3}$, PES-OH and PES-Boc, b. the ${ }^{1} \mathrm{H}-\mathrm{NMR}$ spectrum of CDBMP. 